\title{
REPLICA-BASED CRACK INSPECTION
}

John A. Newman, Stephen W. Smith, and R.S. Piascik NASA Langley Research Center, Hampton, Virginia

Scott A. Willard

Lockheed Martin Space Operations, Hampton Virginia

David S. Dawicke

AS\&M, Hampton, Virginia 


\section{INTRODUCTION}

- Cracks found in Space Shuttle Main Engine $\mathrm{LH}_{2}$ feedline flowliners (2002)

- Ranged from 0.1 inch to 0.6 inch long

- Weld repaired, polished, and recertified for flight

- NDE: no cracks >0.075 inches long exist

- Revisited in 2004

- Unable to show flight rationale with a crack 0.075 inches long 


\section{FLOWLINER DESCRIPTION}

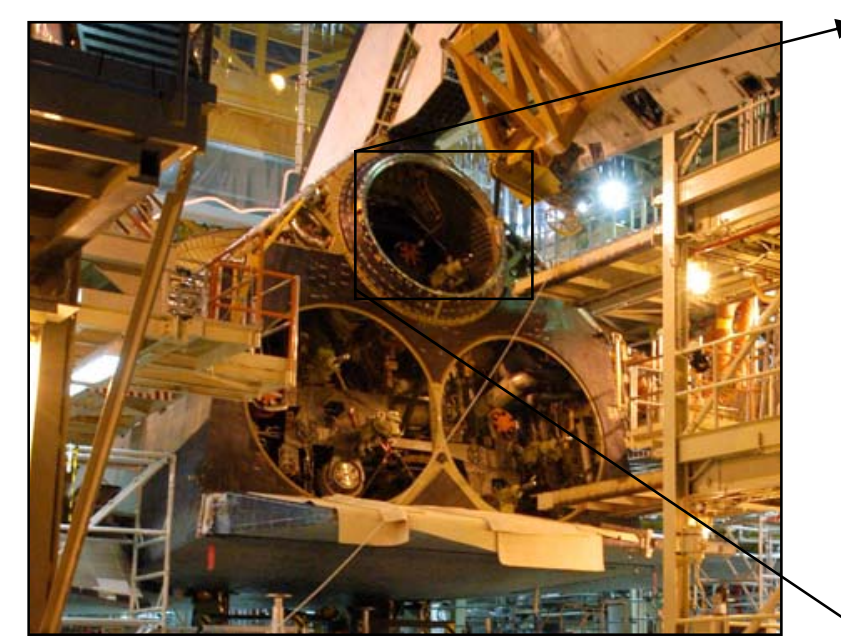

Orbiter aft

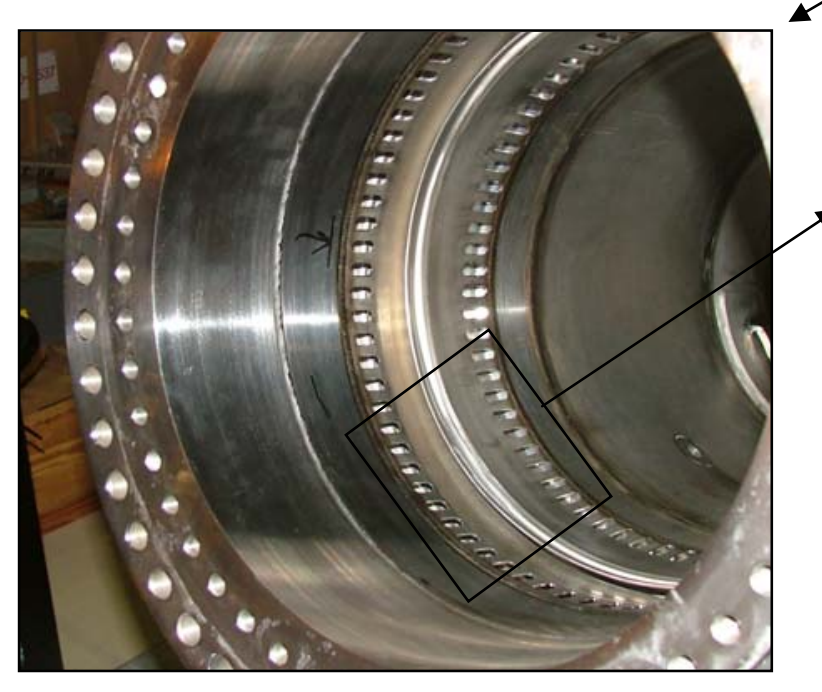

$\mathrm{LH}_{2}$ feedline

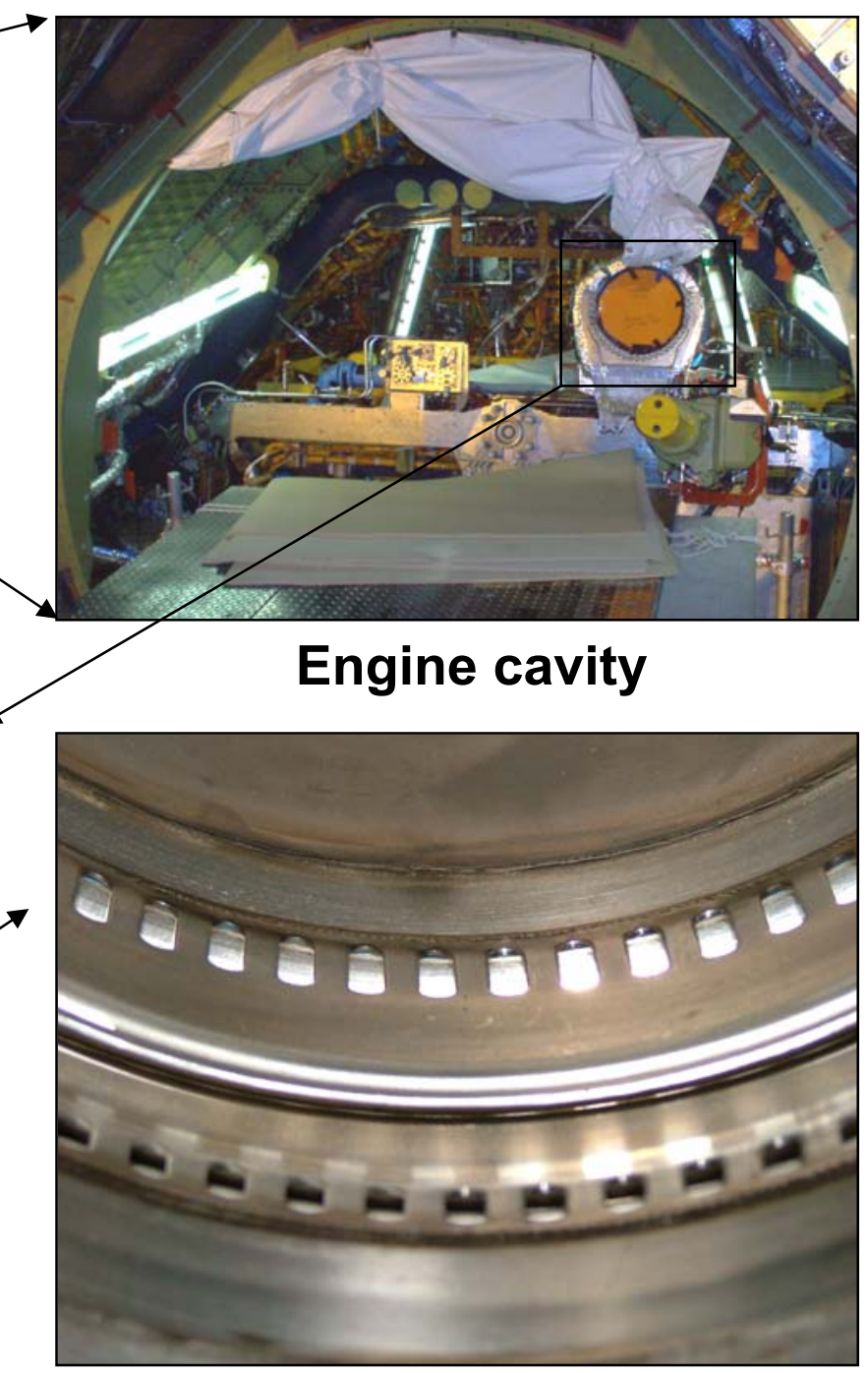

Flowliners 


\section{FLOWLINER DESCRIPTION}

- $\mathrm{LH}_{2}$ consumption

$-385,000$ gallons

- 8.5 minutes

- Each engine consumes 15,000 gal/min

- Flow induced stress cycles in $\mathrm{kHz}$ range

- Millions of stress cycles per flight
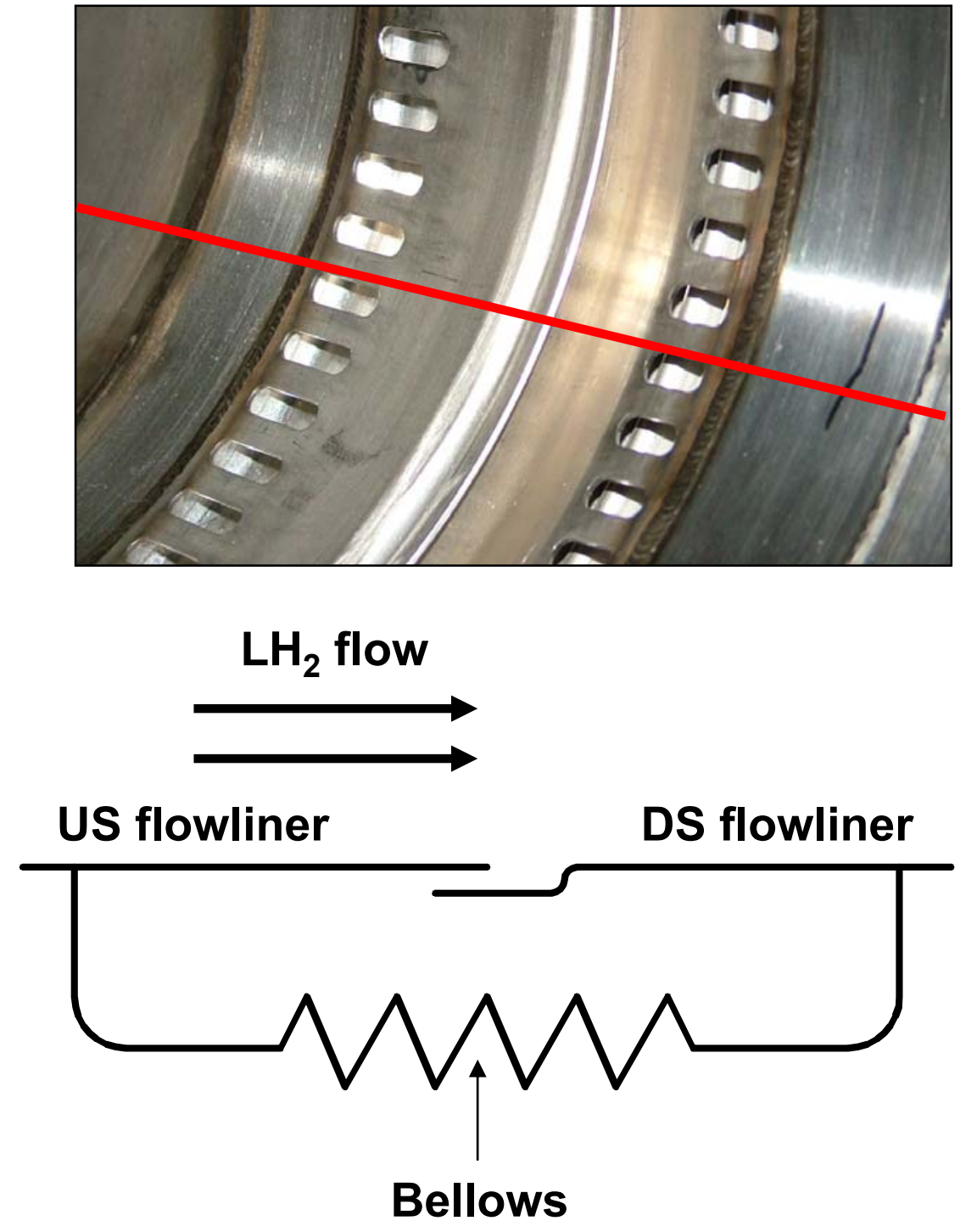


\section{PROBLEM}

- Analysis: unsafe conditions may occur for multiple cracks $>0.005$ inch long

- Improved eddy current unable to detect 0.005-inch-long cracks

- Need an NDE method able to find cracks down to 0.005 inch long 


\section{PROPOSED SOLUTION}

- Use surface replicas as an NDE method

- Surface replicas used for decades to monitor small cracks ( $<0.005$ inch)

- Recently-developed silicone-based replicas better suited for inspection

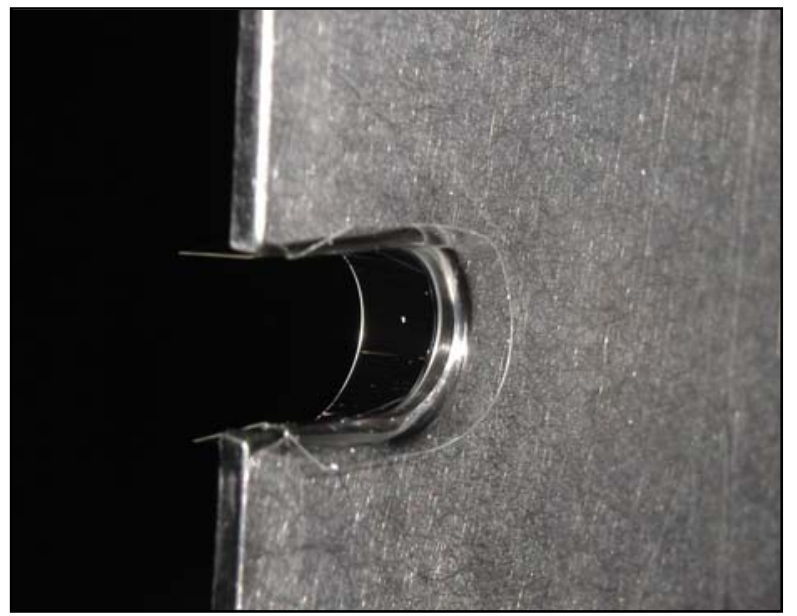

Acetate tape replica

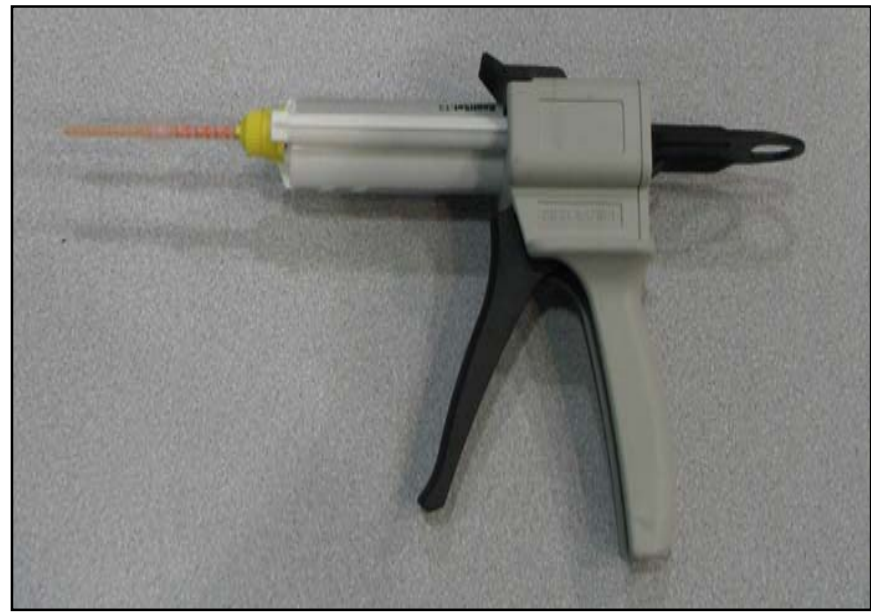

Silicone-based replica dispenser 


\section{EXPERIMENTAL PLAN}

- Feasibility study:

- Generate fatigue cracks in laboratory specimens

- Compare crack lengths from

- Silicone-based replicas (zero load)

- Acetate-tape replicas (maximum load)

- Destructive exam (zero load)

- Determine reliability of siliconebased replicas relative to acetate-tape replicas

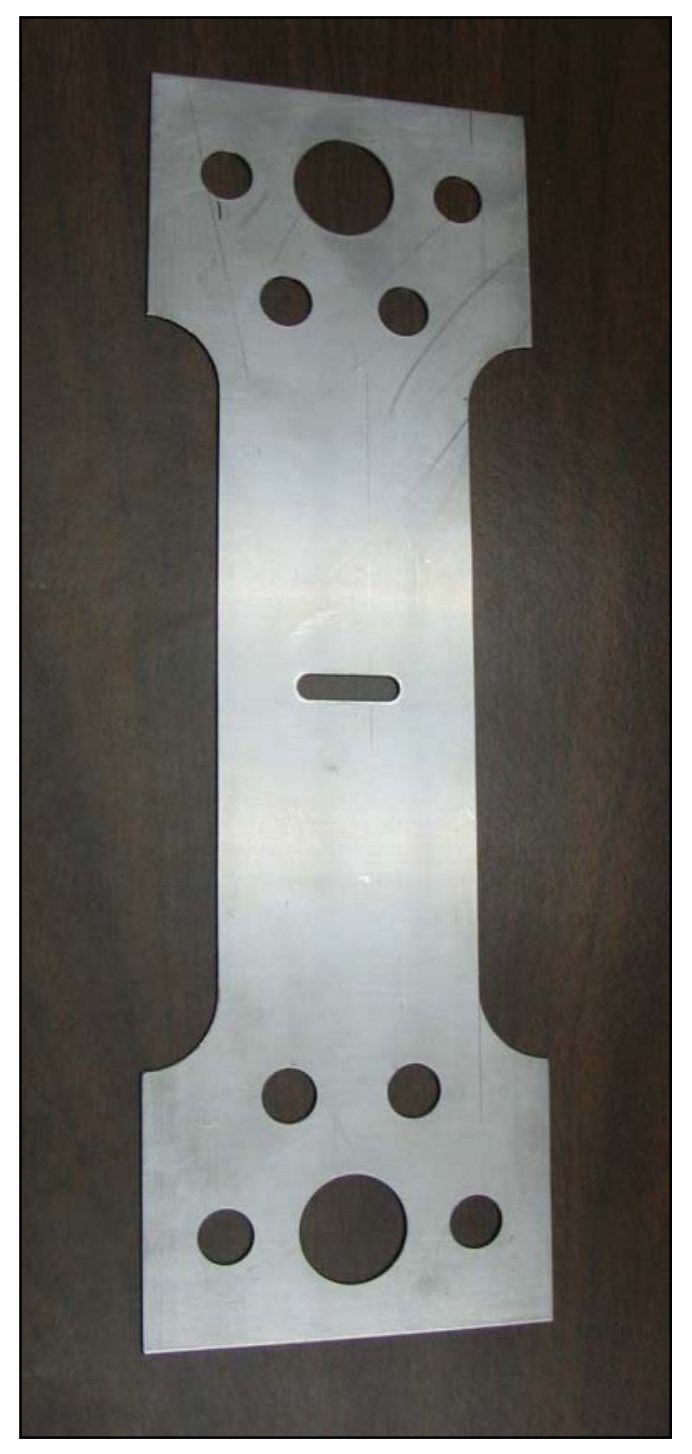




\section{FATIGUE TESTING}

- Specimens used to simulate flowliner slot geometry and stress state

- Pmax = 3.4 kips, $R=0.1$

- Testing interrupted periodically for slot surface replication

- Acetate-tape replicas

- Silicone-based replicas
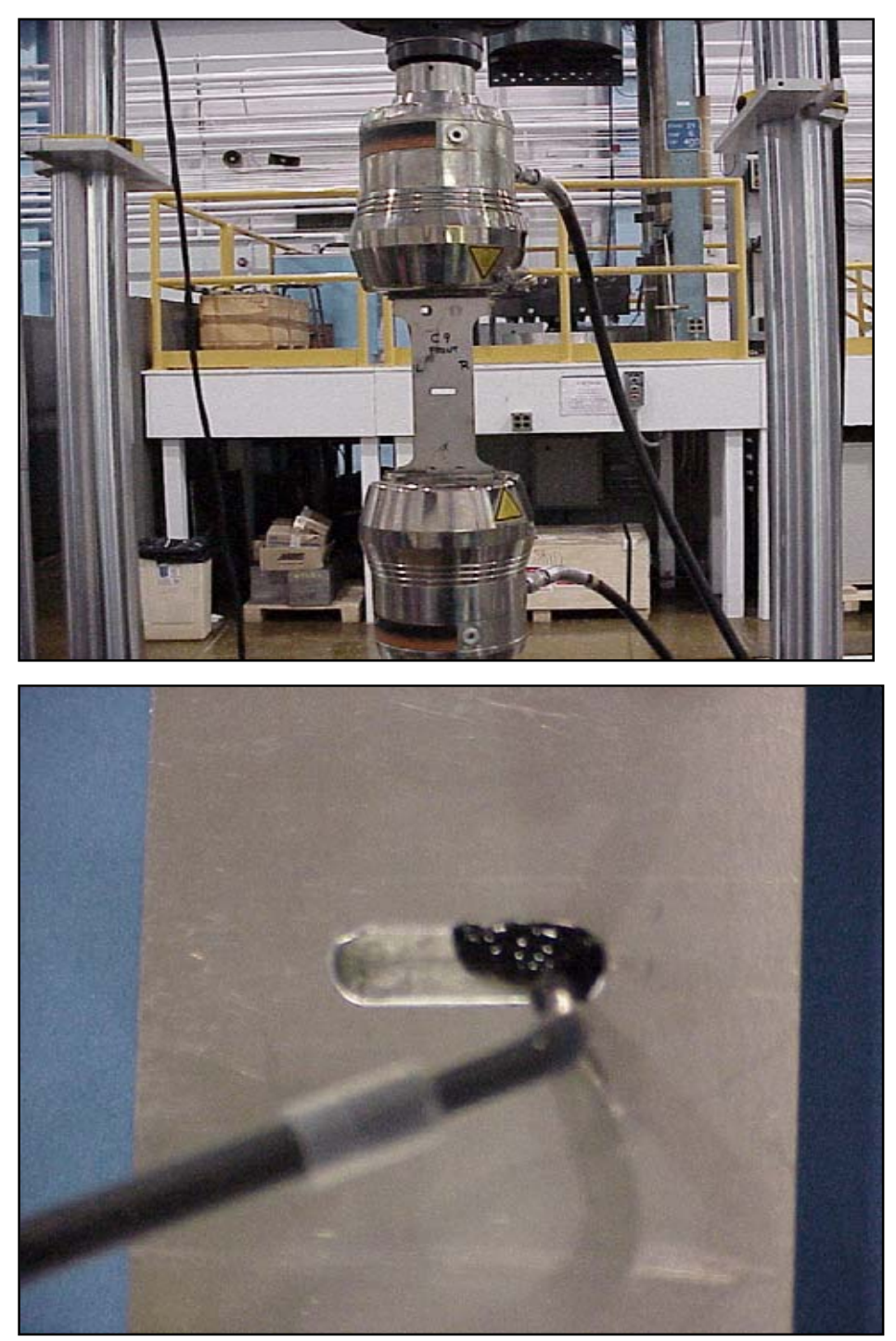


\section{REPLICA ANALYSIS}

- Replica preparation

- Sectioned in 4 pieces

- Grounded on metallic slide

- Coated with metallic material

- Examined in an SEM

- Initial scan at 50-100X

- Surface finish, scratches, etc.

- Crack scan at 400-700X

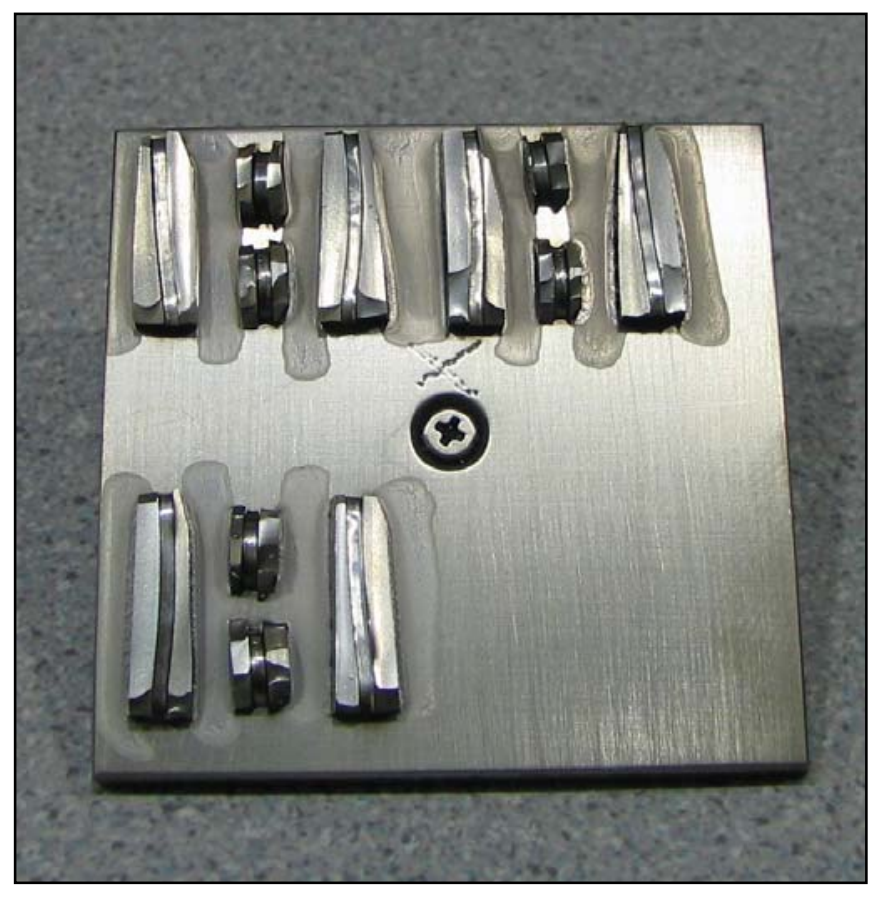




\section{EXPERIMENTAL RESULTS}

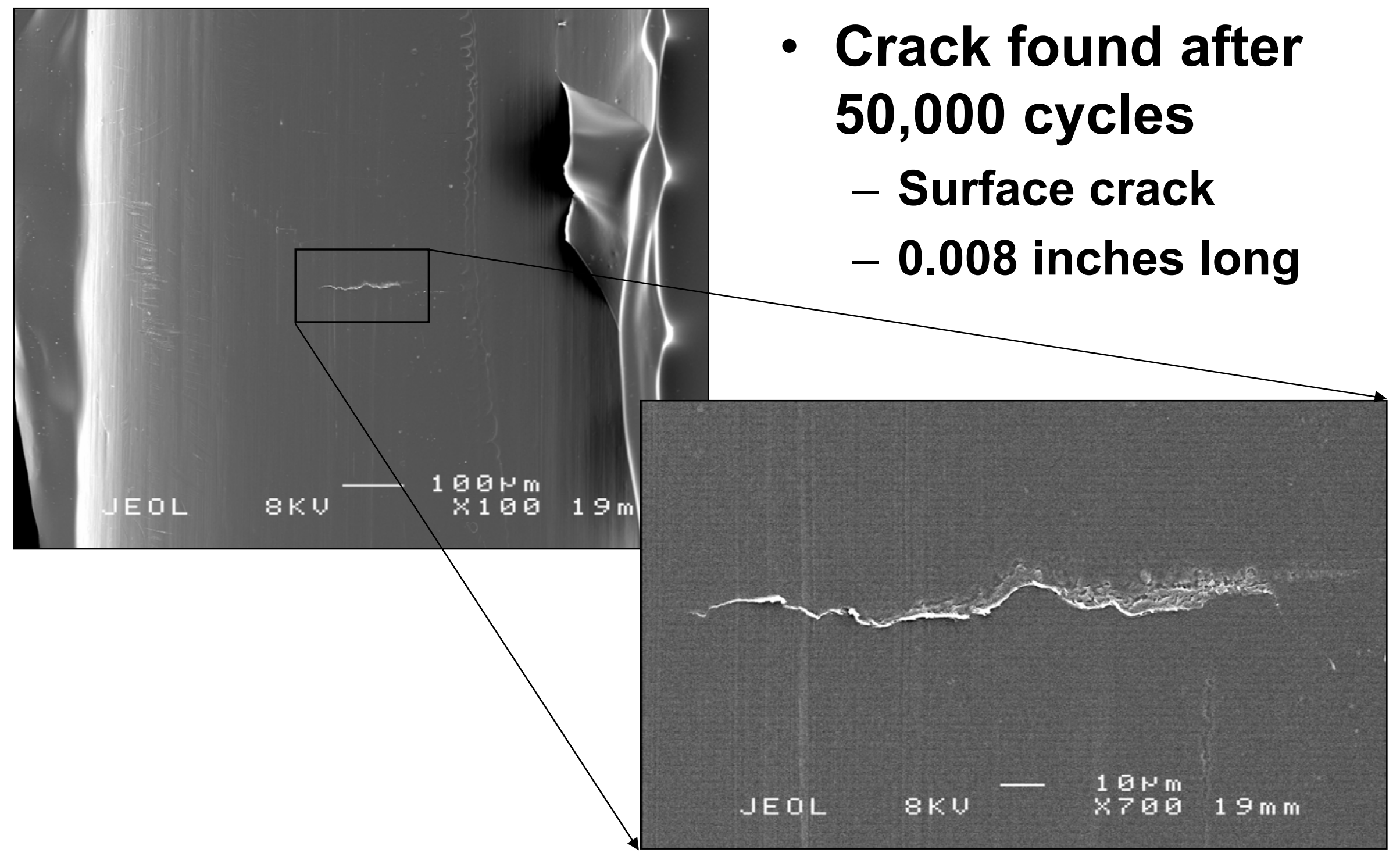




\section{CRACK LENGTH COMPARISON}

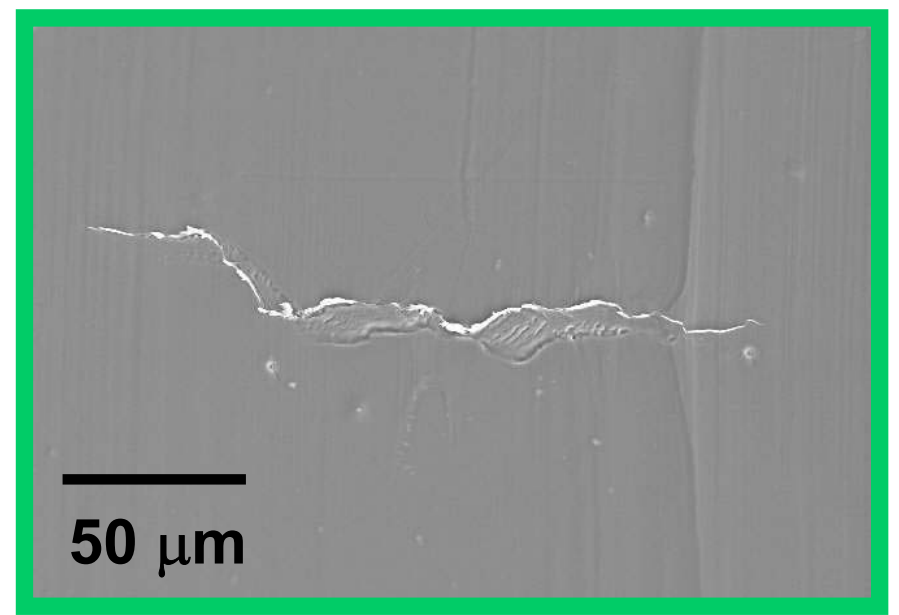

Acetate replica (loaded) - $163 \mu \mathrm{m}$

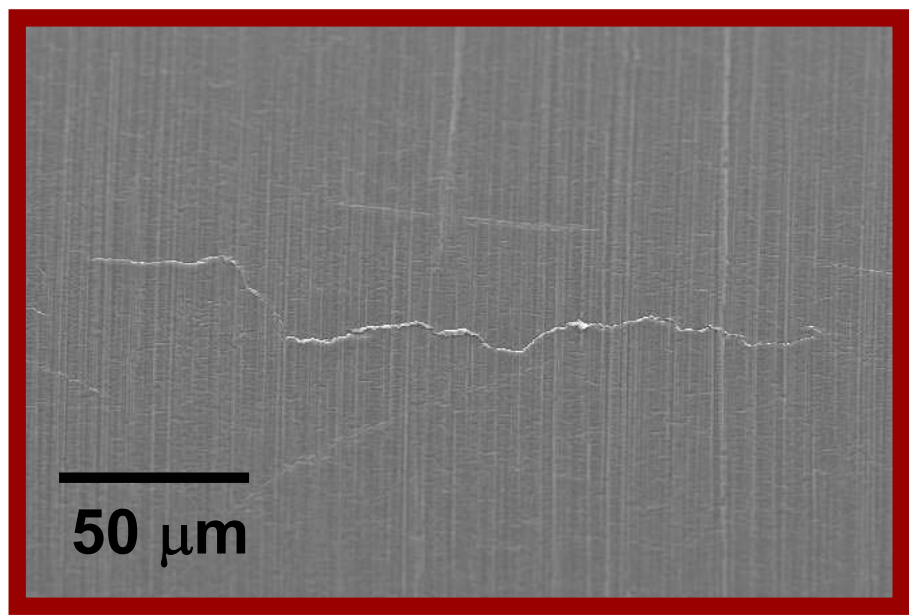

Silicone replica (no load) - $199 \mu \mathrm{m}$

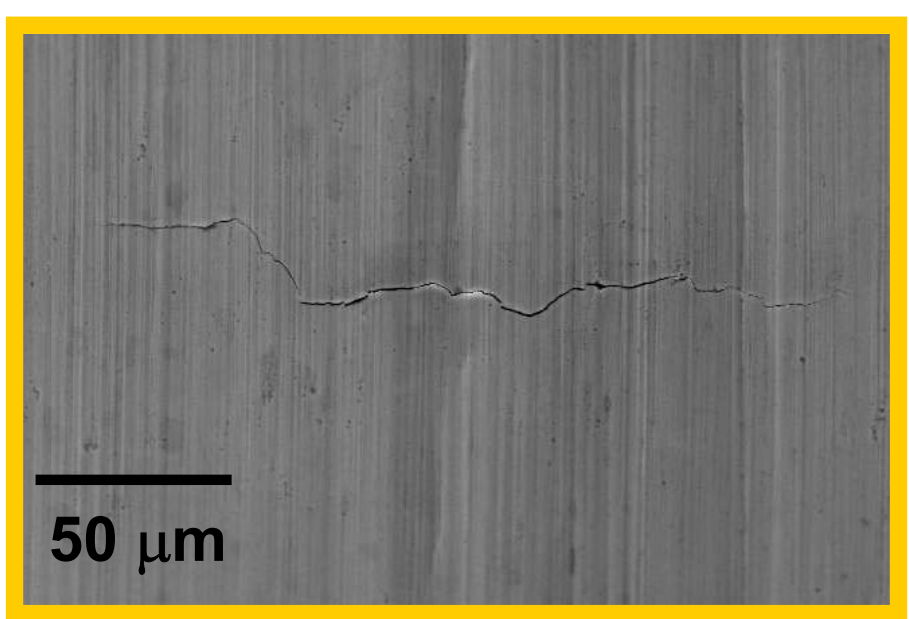

Specimen (no load) - $194 \mu \mathrm{m}$ 


\section{EXPERIMENTAL RESULTS}

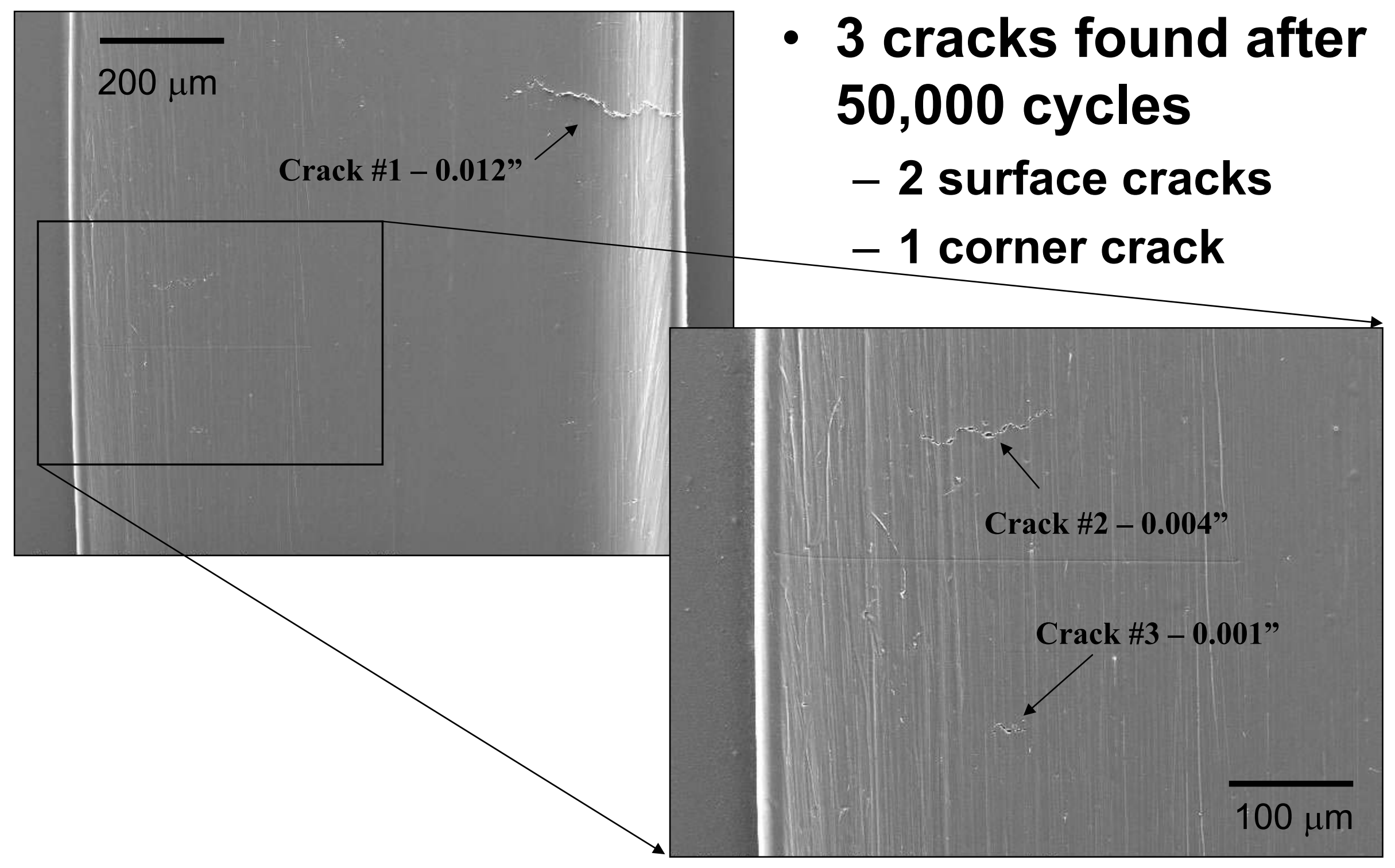




\section{CRACK LENGTH COMPARISON (Crack \#1)}

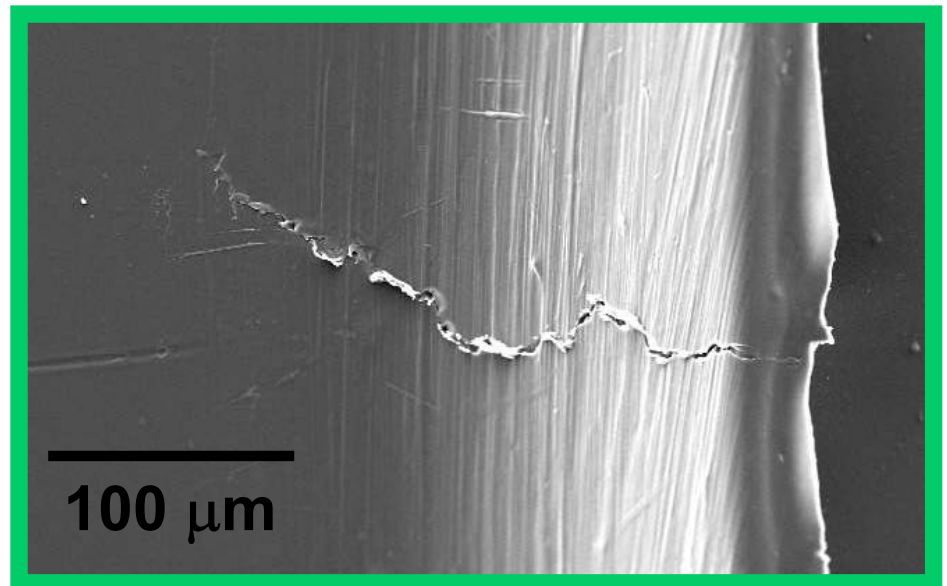

Acetate replica (loaded) $-280 \mu \mathrm{m}$

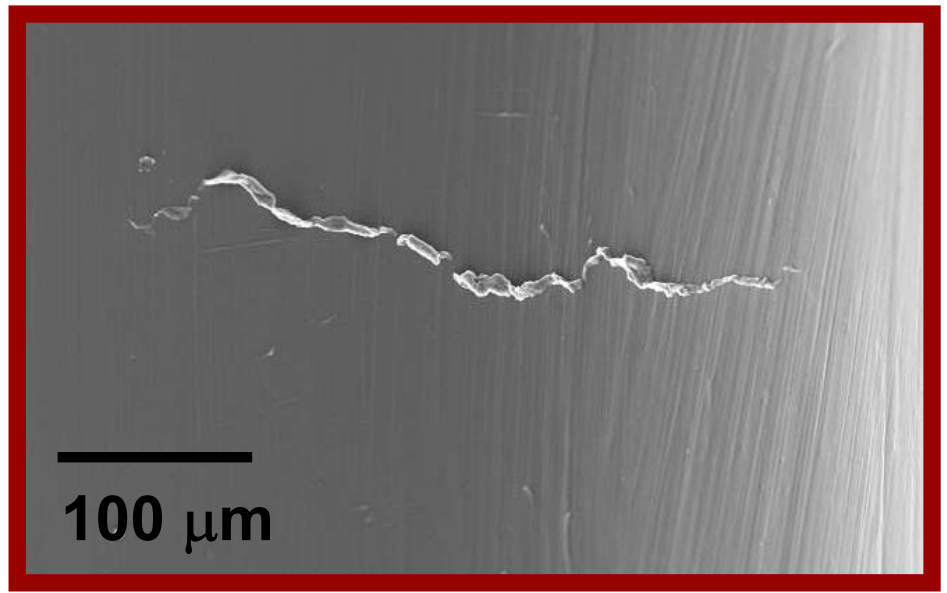

Silicone replica (no load) - $343 \mu \mathrm{m}$

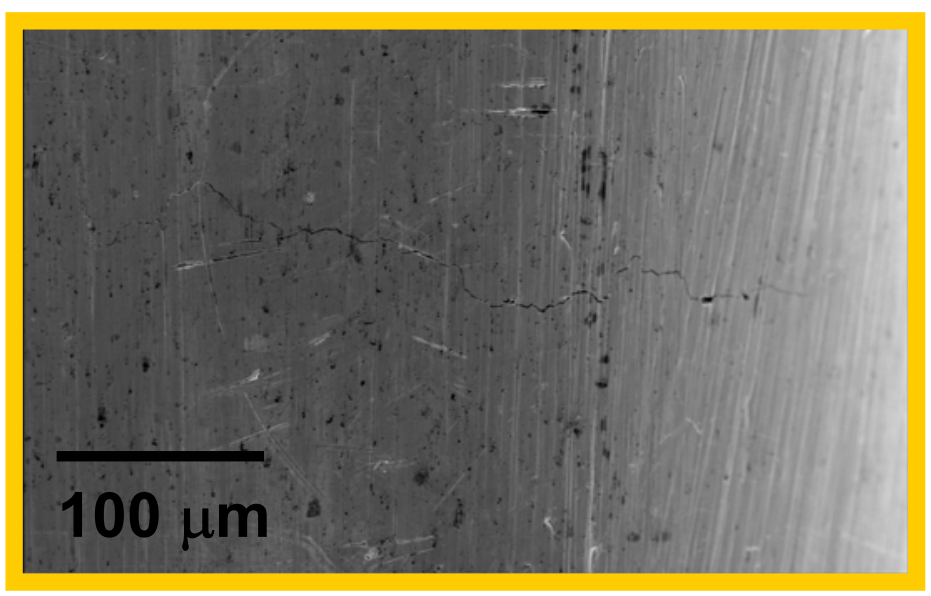

Specimen (no load) $-350 \mu \mathrm{m}$ 


\section{CRACK LENGTH COMPARISON (Crack \#2)}

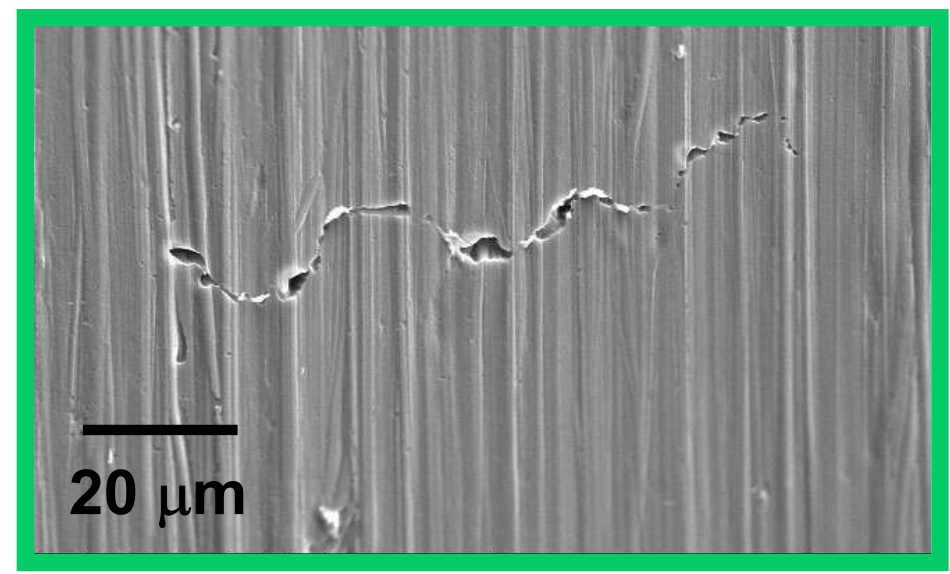

Acetate replica (loaded) $-81 \mu \mathrm{m}$

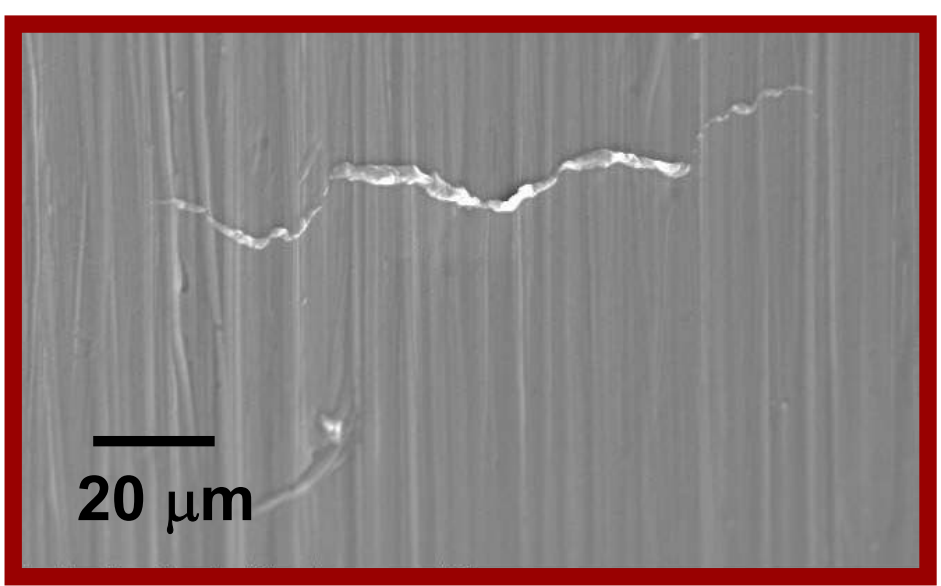

Silicone replica (no load) - $104 \mu \mathrm{m}$

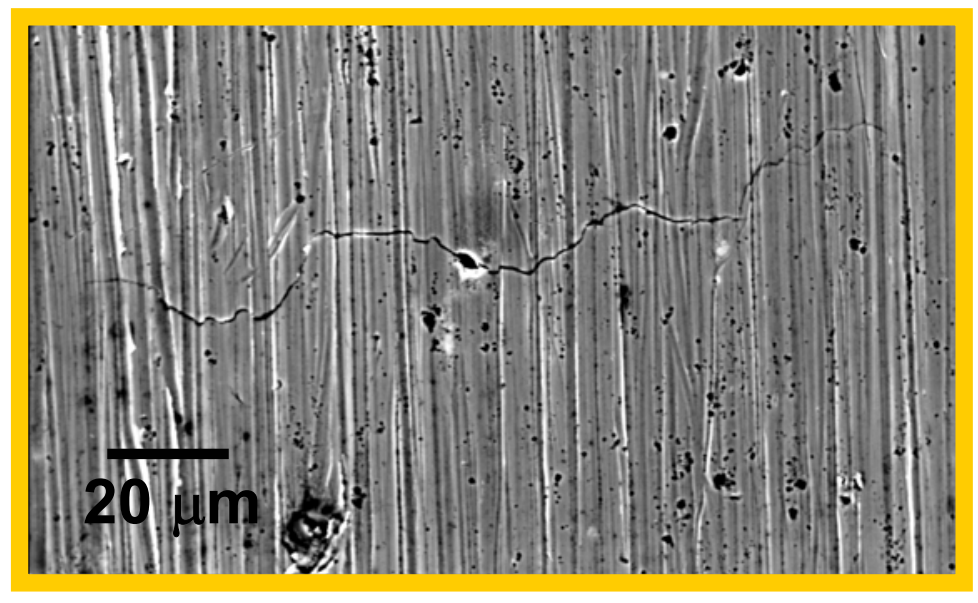

Specimen (no load) - $110 \mu \mathrm{m}$ 


\section{CRACK LENGTH COMPARISON (Crack \#3)}

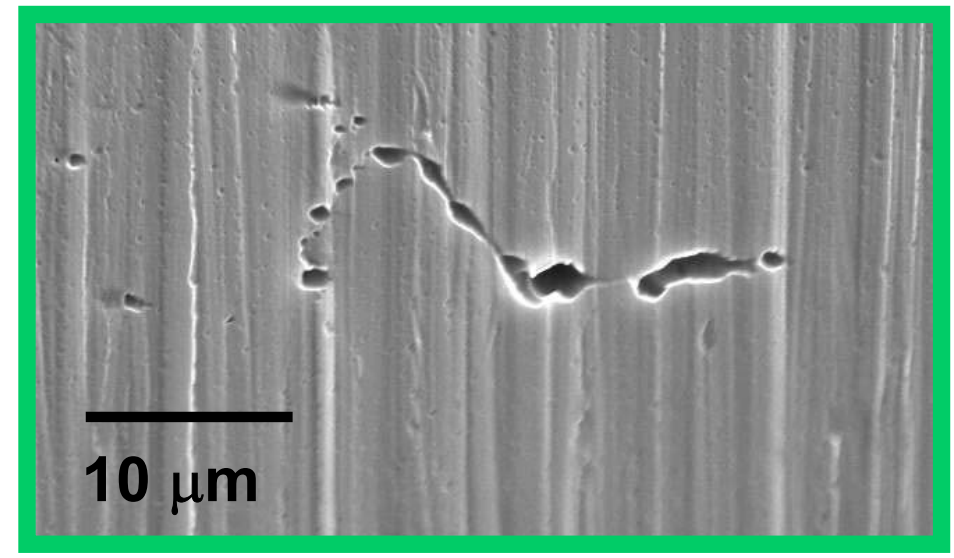

Acetate replica (loaded) $-20 \mu \mathrm{m}$

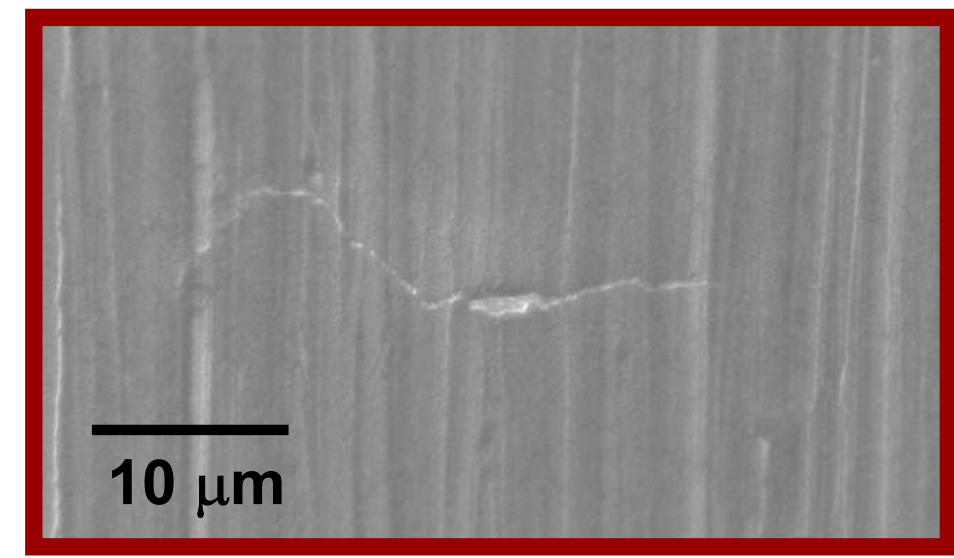

Silicone replica (no load) - $26 \mu \mathrm{m}$

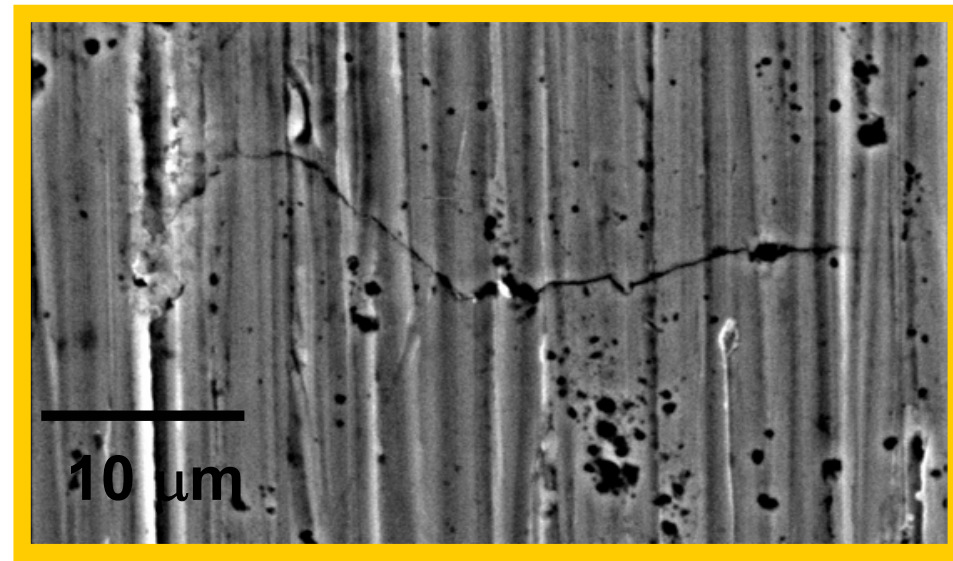

Specimen (no load) - $27 \mu \mathrm{m}$ 


\section{CRACK DETECTION AFTER POLISHING}

- Flowliner slots were polished after cracks detected in 2002

- One orbiter has not flown since flowliner slot polishing

- Concern about post-polishing crack detection

- Crack mouth potentially filled with material 


\section{POLISHED CRACK DETECTION}

Initial crack

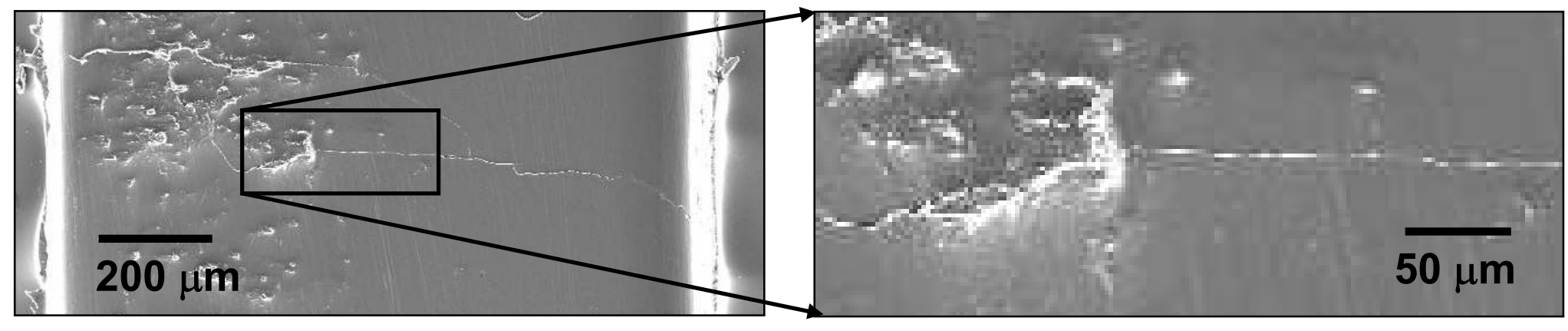

\section{After polishing}

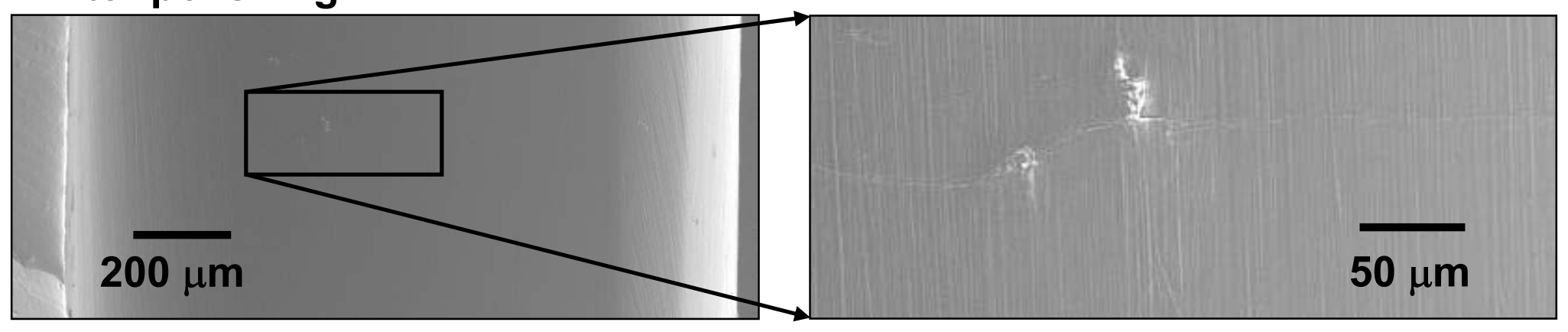

After polishing + 1 load cycle

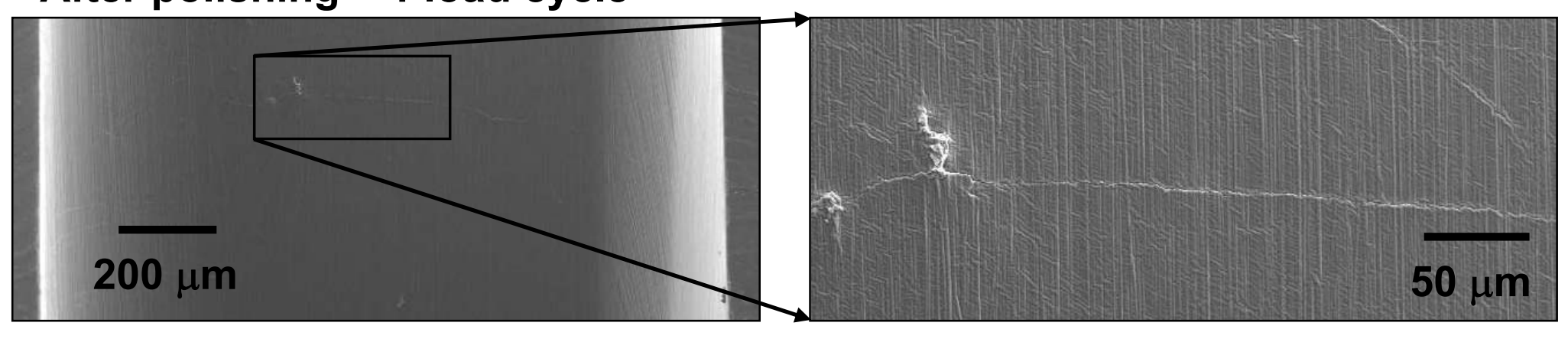




\section{SURFACE FINISH QUALITY}

- Pit-like damage from punching not completely removed by polishing

- At least 7 fatigue cracks initiated by 50,000 cycles

- Quality of surface finish is important

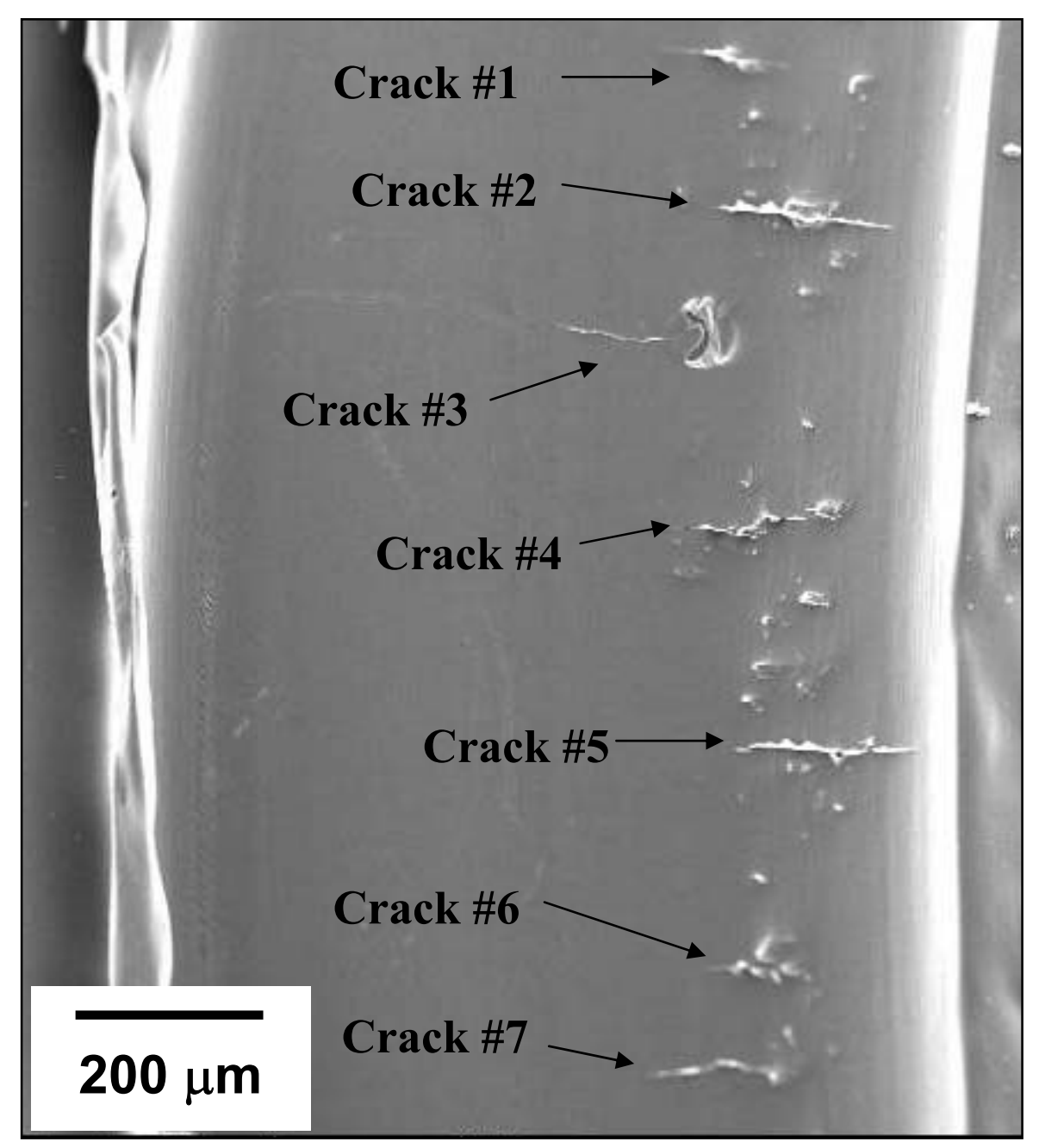




\section{OTHER TYPES OF DAMAGE}

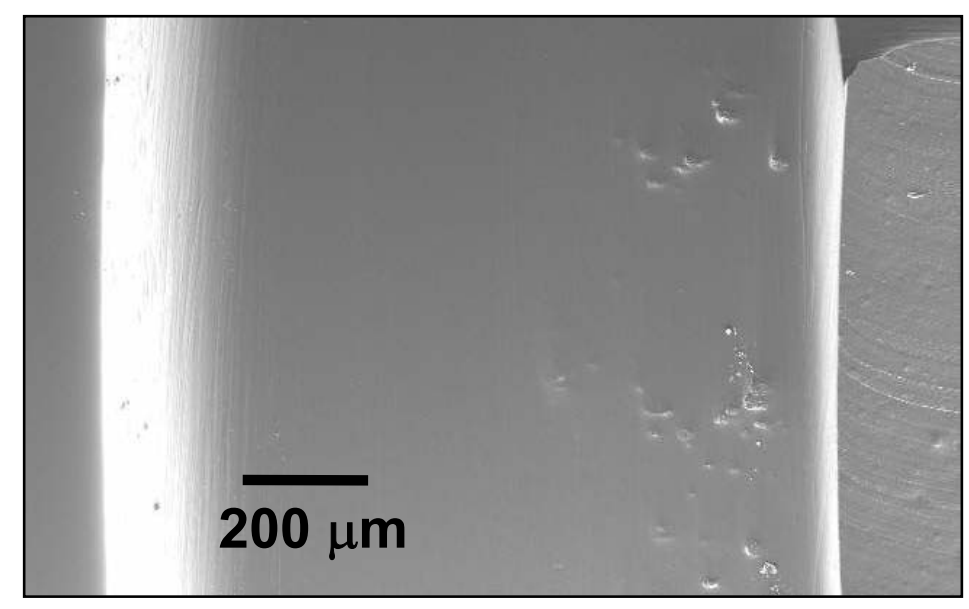

Pit damage

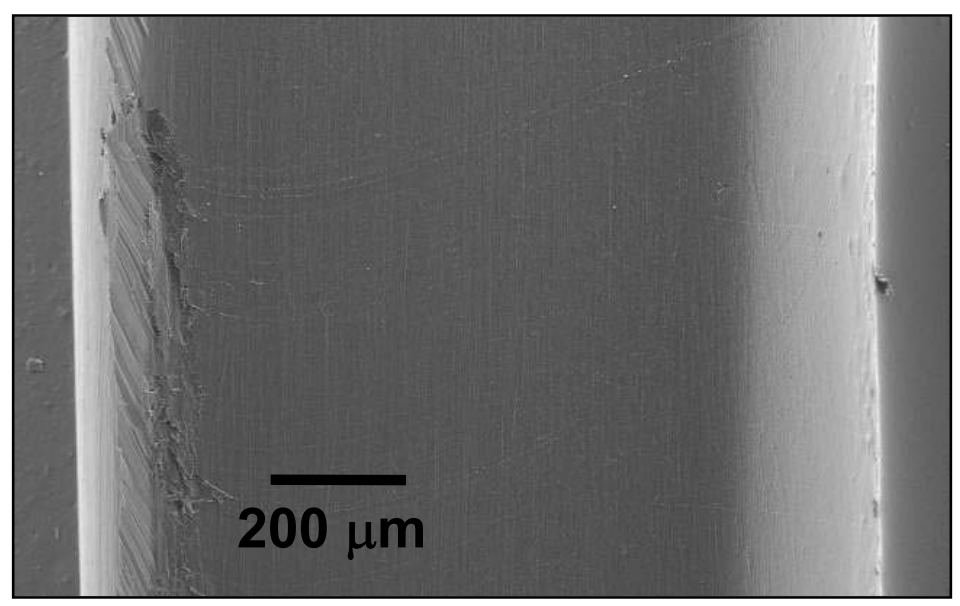

Abrasion and scratches

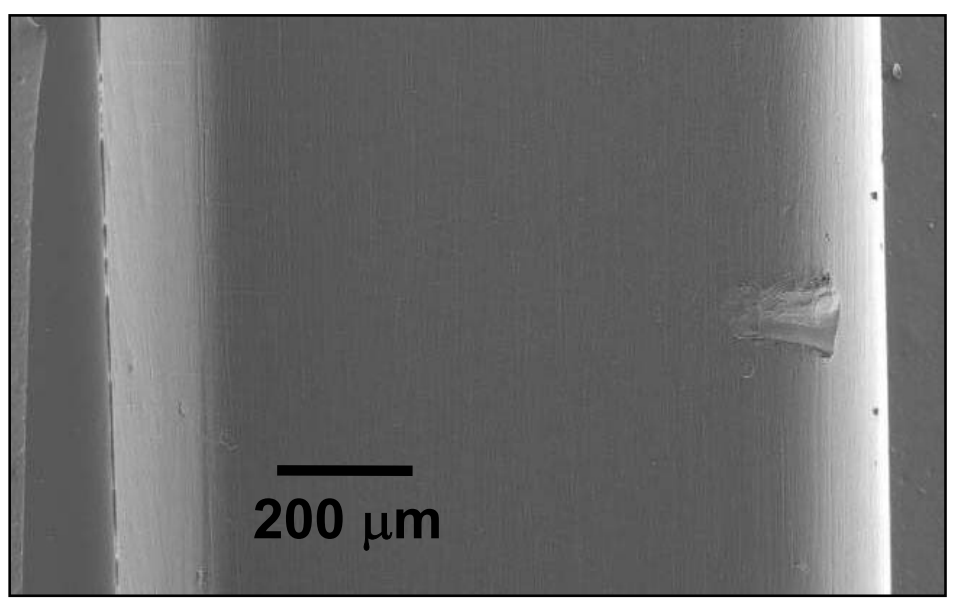

Tool mark

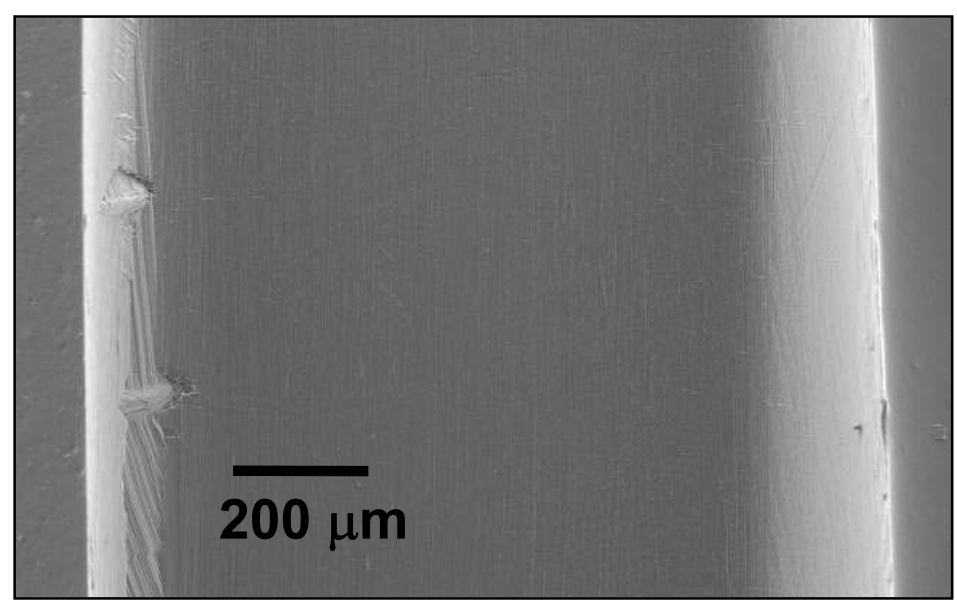

Tool marks/dents 


\section{REPRODUCIBILITY}

- Concern: Repeated replication may fill crack mouth

- Repeated replicas taken on several cracked specimens

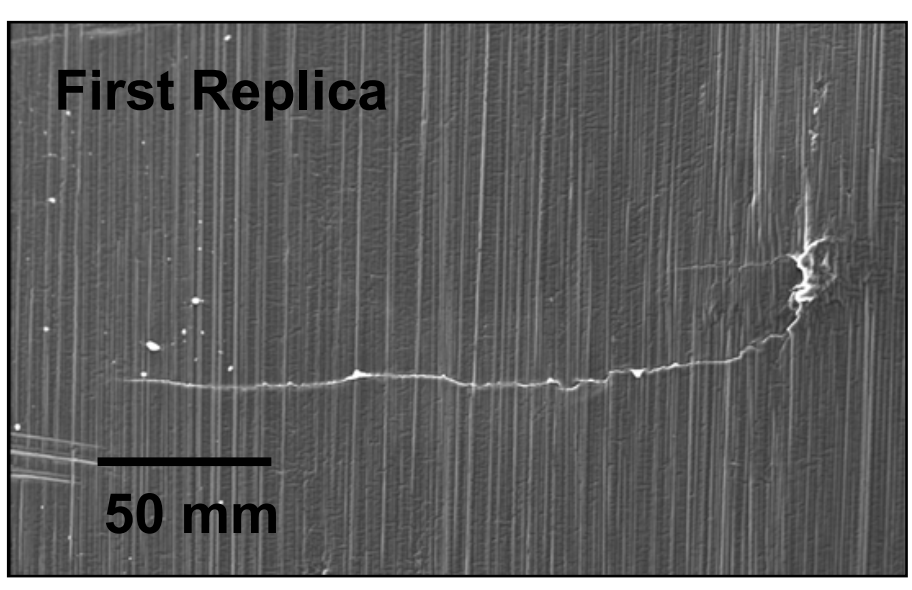

- Example: 0.006-inch-long surface crack

- No degradation in crack detection

Fifth Replica

$50 \mathrm{~mm}$ 


\section{APPLICATION}

- Replica-based inspection method approved for use on flight hardware

- Found 55 cracks in 3 orbiters

- Ranging from 0.004 to 0.040 inches

- Confirmed repair by second round of replicas

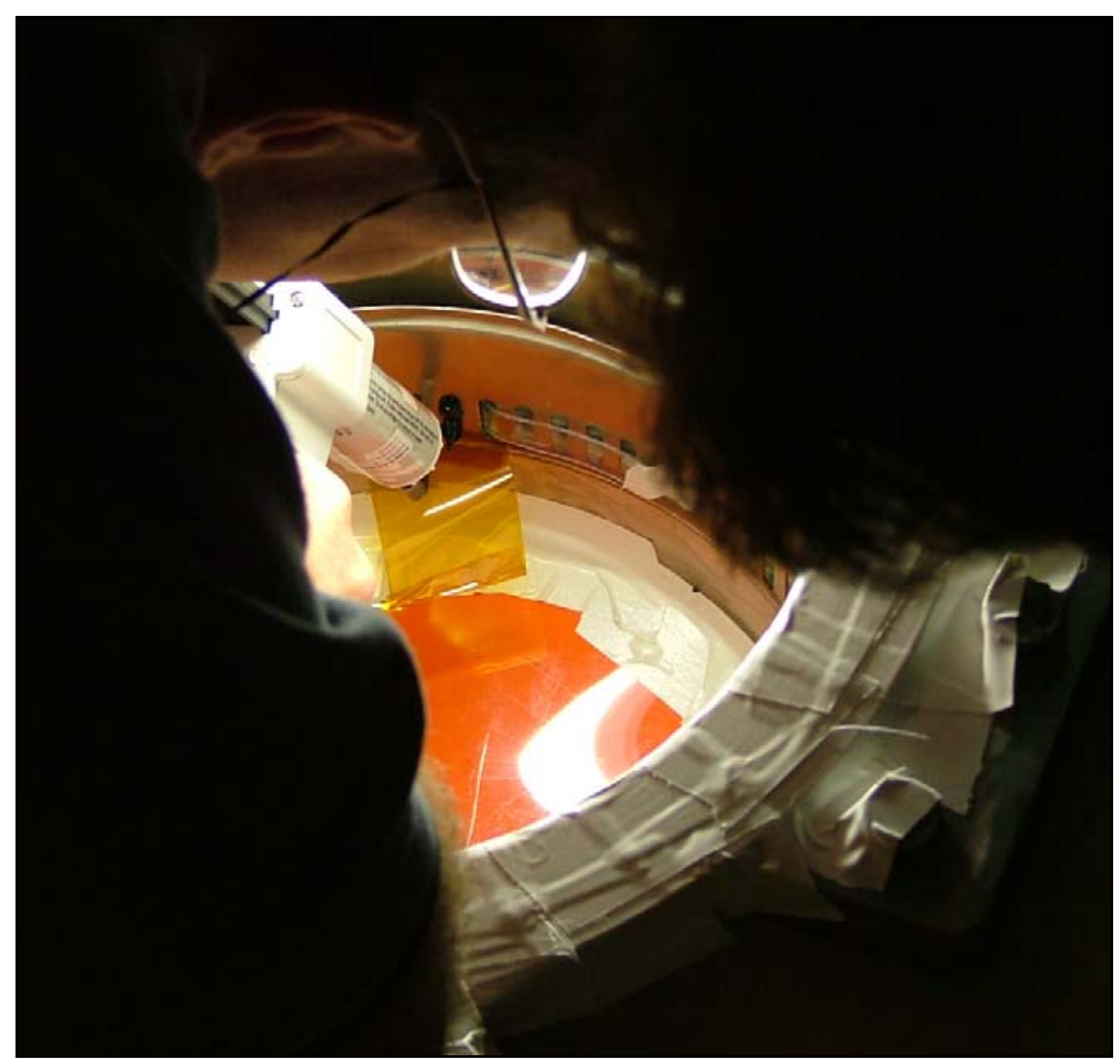




\section{OTHER APPLICATIONS}

- Replica-based crack inspection may be well-suited for other applications

- Improved crack detection could make damage tolerance life management practical for additional components

- Rotorcraft ?

- Propellers?

- HCF engine components? 


\section{PROS AND CONS}

PROS

- Much better resolution than traditional NDE

- Little training required to make replicas

- Limited equipment needed in field
CONS

- More labor intensive than traditional NDE

- Limited to surface flaws

- Dependent on surface condition

- Limited to small areas

- No immediate feedback 


\section{SUMMARY}

- Analysis of silicone-based replicas

- Find cracks below 0.005 inches

- Find pits/defects down to 0.001 inches

- Method approved for use on flight hardware

- Found 55 cracks in 3 orbiters (684 slots)

- Identified unacceptable levels of damage

- Repair confirmed by second round of replicas 